\title{
Can cash help eliminate mother-to-child HIV transmission?
}

See Articles page e85

Great strides have been made in reducing mother-tochild transmission (MTCT) of HIV in recent years. The global plan to eliminate HIV infection in children by 2015 and keep their mothers alive has reduced new HIV infections among children by nearly half since 2009. ${ }^{1}$ Many countries in sub-Saharan Africa, where the epidemic is most severe, have moved to providing combination antiretroviral therapy (ART) to all pregnant women irrespective of $C D 4$ count (known as option $B+$ ), which has resulted in many more women accessing ART and significantly reducing MTCT.,3

Despite the great potential of ART to eliminate MTCT, important challenges remain in both supply (eg, drug availability, clinical staff, infant diagnostic capability) 
and demand (eg, clinic attendance, stigma, treatment adherence). These challenges result in substantial loss to follow-up of pregnant women both before and after delivery. Close to $50 \%$ of HIV infected pregnant women are lost between antenatal clinic registration and delivery ${ }_{i}^{4}$ without retention and adherence interventions, MTCT is common. However, to date there are few evidence-based interventions to improve retention in care for people living with HIV, including pregnant women. ${ }^{5}$

In The Lancet HIV, Marcel Yotebieng and colleagues ${ }^{6}$ report that a modest cash transfer provided to HIV positive pregnant women in Kinshasa, Democratic Republic of the Congo, resulted in more women attending all clinical visits and remaining in care up to 6 weeks post partum. Eliminating leakages in the MTCT cascade will be crucial for the ultimate eradication of the vertical transmission of HIV, and these data are encouraging but also call for a better understanding of contextual and operational issues in the setting of antenatal care especially in very resource constrained regions.

Context is incredibly important in the design and eff ectiveness of cash transfer programmes. The UNAIDS motto of "know your epidemic" is especially relevant for cash transfer programmes: what is the goal of providing cash to individuals? Is there plausible evidence that cash will overcome the barriers to behaviour change in the target population? How much cash is needed to achieve the desired behaviour change? And what are the mechanisms of action through which the cash will effect change?

The logistical challenges of conditional cash transfer programmes must not be underestimated. Is it feasible and sustainable to hand out cash in clinical settings? What systems need to be in place to prevent theft or corruption? How will how will conditions being met be monitored? Significant staff and infrastructure are required for these steps.

Other important programmatic considerations include who to provide cash to, how long to provide it for, and how best to provide cash in combination with other prevention and care services. If cash transfers for prevention of MTCT are considered, programmes might target those at greatest risk of loss to follow-up. In the study by Yotebieng and colleagues, ${ }^{6}$ the eff ects were greatest in the most vulnerable population-those in lowest wealth quintile, those who had to walk to the clinic, and those who had not disclosed their HIV status. In terms of length of provision of cash, prevention of MTCT is a defined period of time. In view of the concern about HIV transmission post partum and while breastfeeding, cash transfer programmes might include interventions during this time as well. A related question is whether and how to combine other promising interventions, such as peer-based or male engagement interventions, $^{7-9}$ with cash transfer interventions for optimum benefit. The study reported here noted that retention in this programme had already significantly improved before cash being introduced, emphasising the effect that basic health system strengthening can have and reminding us that cash alone will not be enough to achieve our goal of elimination of MTCT.

The study by Yotebieng and colleagues ${ }^{6}$ adds to a body of evidence showing that small incentives can improve uptake or retention in health services-some of these services have included male circumcision, HIV test results, antenatal clinic services, and childhood immunisations. ${ }^{10-13}$ We are at a cross roads in terms of the AIDS response. If we are to meet the ambitious but exciting prevention targets set by UNAIDS and other agencies in the years ahead, we will need to consider every potential intervention that might facilitate those goals. Cash incentives are a viable intervention for further scrutiny and deployment.

\section{${ }^{*}$ Audrey Pettifor, Nora Rosenberg, Linda-Gail Bekker} Department of Epidemiology, University of North Carolina at Chapel Hill, Chapel Hill, NC 27599, USA (AP); School of Public Health and Medicine, University of the Witwatersrand, Johannesburg, South Africa (AP); Institute for Global Health and Infectious Diseases, University of North Carolina at Chapel Hill, Chapel Hill, NC, USA (NR); and The Desmond Tutu HIV Centre, University of Cape Town, Cape Town, South Africa (L-GB)

apettif@email.unc.edu

We declare no competing interests.

1 UNAIDS. Global Plan towards the elimination of new HIV infections among children by 2015 and keeping their mothers alive. Geneva: Joint United Nations Programme on HIV/AIDS, 2011.

2 Ahmed S, Kim MH, Abrams EJ. Risks and benefits of lifelong antiretroviral treatment for pregnant and breastfeeding women: a review of the evidence for the Option B+ approach. Curr Opin HIV AIDS 2013; 8: 474-89.

3 Centers for Disease Control and Prevention. Impact of an innovative approach to prevent mother-to-child transmission of HIV-Malawi, July 2011-September 2012. MMWR Morb Mortal Wkly Rep 2013; 62: 148-51.

4 Sibanda EL, Weller IV, Hakim JG, Cowan FM. The magnitude of loss to follow-up of HIV-exposed infants along the prevention of mother-to-child HIV transmission continuum of care: a systematic review and meta-analysis. AIDS 2013; 27: 2787-97.

5 Rollins N, Chanza H, Chimbwandira F, et al. Prioritizing the PMTCT implementation research agenda in 3 African countries: INtegrating and Scaling up PMTCT through Implementation REsearch (INSPIRE) J Acquir Immune Defic Syndr 2014; 67 (suppl 2): S108-13.

6 Yotebieng $\mathrm{M}$, Thirumurthy $\mathrm{H}$, Moracco KE, et al. Conditional cash transfers and uptake of and retention in prevention of mother-to-child HIV transmission care: a randomised controlled trial. Lancet HIV 2016; 3: 85-93. 
7 Rosenberg NE, Mtande TK, Saidi F, et al. Recruiting male partners for couple HIV testing and counselling in Malawi's option B+ programme: an unblinded randomised controlled trial. Lancet HIV 2015; 2: e483-91.

8 Gourlay A, Birdthistle I, Mburu G, lorpenda K, Wringe A. Barriers and facilitating factors to the uptake of antiretroviral drugs for prevention of mother-to-child transmission of HIV in sub-Saharan Africa: a systematic review. J Int AIDS Soc 2013; 16: 18588.

9 Ahmed S, Kim MH, Dave AC, et al. Improved identification and enrolment into care of HIV-exposed and -infected infants and children following a community health worker intervention in Lilongwe, Malawi. J Int AIDS Soc 2015; 18: 19305

10 Thirumurthy $\mathrm{H}, \mathrm{Masters} \mathrm{SH}$, Rao S, et al. Effect of providing conditional economic compensation on uptake of voluntary medical male circumcision in Kenya: a randomized clinical trial. JAMA 2014; 312: 703-11.
11 Lagarde M, Haines A, Palmer N. Conditional cash transfers for improving uptake of health interventions in low- and middle-income countries: a systematic review. JAMA 2007; 298: 1900-10.

12 Barham T, Maluccio JA. Eradicating diseases: the effect of conditional cash transfers on vaccination coverage in rural Nicaragua. J Health Econ 2009; 28: 611-21.

13 Thornton R. The demand for, and Impact of, learning HIV Status. Am Econ Rev 2008; 98: 1829-63. 